\title{
A continuidade da execução dos processos de software em empresas avaliadas no MPS.BR
}

\author{
Carlos Diego A. Almeida ${ }^{1}$, Thiago C. Macedo ${ }^{1}$, Adriano B. Albuquerque ${ }^{1}$ \\ ${ }^{1}$ UNIFOR - Universidade de Fortaleza \\ Fortaleza-CE - Brazil \\ \{carlosdiegoa, crystyanjlc\}@gmail.com, adrianoba@unifor.br
}

\begin{abstract}
Resumo. Muitas empresas desenvolvedoras de software encontram, nos modelos de maturidade, uma forma de melhorar a qualidade de seus produtos. Os modelos utilizam-se de avaliações para qualificar essas empresas, que investem recursos na definição e na implementação de seus processos. Entretanto, após a avaliação, em alguns casos, as empresas percebem dificuldades em manter seus processos aderentes ao modelo adotado. Esse artigo apresenta os resultados de uma pesquisa quantitativa e de uma qualitativa que buscaram capturar e representar os conhecimentos relacionados aos fatores que influenciam a continuidade da execução dos processos de software de forma aderente ao modelo MPS.BR.
\end{abstract}

Abstract. Many software development companies find in the maturity models, one way to improve the quality of their products. The models make use of assessments to qualify those companies. The companies invest resources in definition and implementation of their processes. However, after an assessment, in some cases, companies realize difficulties to maintain their processes compliant with the adopted model. This article presents the results of a quantitative research and a qualitative research which tried to capture and represent the knowledge related to the factors that influence in the maintenance of execution of software processes in a manner compliant to the model MPS.BR.

\section{Introdução}

A qualidade tornou-se um dos principais fatores de destaque no mercado atual. A procura pela excelência e pela confiabilidade dos produtos gerados é um objetivo a ser alcançado pelas organizações, a fim de permanecerem atuantes nesse cenário competitivo. Na globalização do mercado de software, terá mais chance de sobreviver quem for organizado e eficiente no seu processo de produção, disponibilização e evolução de software (Pressman, 2006).

Alcançar competitividade pela qualidade, para as empresas de software, implica tanto na melhoria da qualidade dos produtos de software e serviços correlatos, como dos processos de produção e distribuição de software (SOFTEX, 2009).

Diante da complexidade e da especificidade dos processos de software, que são influenciados por fatores técnicos, culturais e ambientais, foram realizadas várias iniciativas para definir modelos e normas que pudessem guiar as organizações a desenvolverem seus softwares. Os que mais se destacam são a norma ISO/IEC 12207 (ISO/IEC, 2004), a norma ISO/IEC 15504 (ISO/IEC, 2002), o MPS.BR (SOFTEX, 2009) e o CMMI (CMU/SEI, 2006). 
Dentre esses, um modelo que se destaca no Brasil é o MPS.BR. De acordo com SOFTEX (2009), o programa MPS.BR, lançado em dezembro de 2003, visa à melhoria dos processos de software das micro, pequenas e médias empresas brasileiras. Entretanto, como outros modelos de maturidade, após a implantação, constatam-se problemas em manter o processo aderente ao modelo de referência. Dessa forma, é importante saber as principais dificuldades em manter o processo aderente ao modelo.

Esse artigo apresenta os resultados de uma revisão bibliográfica, com o objetivo de encontrar quais fatores influenciam na manutenção dos processos aderentes ao MPS.BR, e também apresenta uma pesquisa quantitativa e uma pesquisa qualitativa em que se procurou capturar e representar os conhecimentos relacionados com esses fatores.

Esse artigo está organizado em cinco seções: a seção 1 consiste na introdução, a seção 2 apresenta uma revisão bibliográfica, a seção 3 expõe a metodologia e os resultados obtidos do survey com os profissionais envolvidos com o modelo MPS.BR, a seção 4 mostra a metodologia de pesquisa qualitativa baseada na Grounded Theory e os resultados de sua aplicação; e, por fim, a seção 5 contém a conclusão do artigo e a apresentação de futuros trabalhos.

\section{Revisão Bibliográfica}

Para a revisão da literatura, foram utilizados alguns passos de uma revisão sistemática, um tipo de estudo secundário, difundido por Kitchenham (2004), a fim de identificar fatores que podem influenciar a continuidade da execução dos processos de software. Foi estabelecido um protocolo para a implementação de uma revisão sistemática com base no protocolo desenvolvido por Silva Filho (2006). Também foram usadas ferramentas, como planilhas, para apoio ao registro e à análise dos dados coletados.

O foco da revisão foram os fatores que influenciam a manutenção do processo de qualidade de software, mas se encontrou pouco material falando especificamente sobre essa manutenção. Então, também foram analisados os fatores que relacionavam-se com a implementação de um modelo de melhoria. No entanto, só foram considerados aqueles que se relacionavam com o contexto de continuidade do processo de software.

O primeiro passo da revisão foi buscar todos os artigos publicados em simpósios e workshops pertinentes ao modelo MPS.BR com os termos "manutenção" e "modelo de maturidade", além de buscar artigos em bibliotecas digitais internacionais, como ACM e IEEE. Nessa fase foram identificados trinta artigos.

$\mathrm{Na}$ segunda fase da revisão, excluíram-se os artigos que não faziam menção ao modelo de maturidade MPS.BR e à experiência de manutenção ou implementação desse modelo. Após essa fase, restaram doze artigos.

A terceira fase consistiu na identificação dos artigos que possuíam os fatores pesquisados. Foram encontrados oito artigos que atenderam a todas essas restrições. Os fatores encontrados nesses artigos estão listados abaixo.

De acordo com Yoshida e Tavares (2008), Silva Filho e Katsurayama (2008), Parente e Albuquerque (2008) e Zanetti (2008), o nível de formalização das estruturas organizacionais é um fator que pode facilitar a implementação de um modelo de melhoria de processo. 
Por exemplo, Yoshida e Tavares (2008) observaram que a formalização das estruturas organizacionais em áreas, funções, competências e responsabilidades demonstram um nível de organização que permite uma forma simples de visualizar como a empresa está organizada para atender a seus objetivos. A formalização não deve ser entendida, neste caso, como uma burocracia, mas como um meio para facilitar a comunicação dentro da empresa. Quão maior o nível de organização e formalização da estrutura organizacional, menor é o risco da visão de curto prazo sobre a implementação de melhoria de processos. Esse fator também pode influenciar, positivamente, a manutenção da qualidade, porque uma empresa com uma estrutura organizacional mais formal é capaz, facilmente, de manter a consciência de seus papéis, responsabilidades e áreas.

Também se destacou o nível de ferramentas e técnicas para a reutilização do conhecimento. Parente e Albuquerque (2008), Silva Filho e Katsurayama (2008), Monteiro et al. (2008) e Zanetti (2008) disseram que uma empresa que queira melhorar a maturidade de seus processos deve aproveitar o conhecimento existente na organização. O conhecimento pode ser apoiado por um ambiente de gestão, e, a partir da reutilização do conhecimento, a organização pode implementar melhorias para os processos de software e acelerar a implementação e a execução dos mesmos.

Esse fator também é relevante para o contexto de continuidade, porque a empresa que possui ferramentas e técnicas para apoiar a reutilização do conhecimento pode, com uma maior facilidade, manter vivo o conhecimento do processo implementado, incentivando sua utilização e mantendo clara a necessidade de suas atividades e tarefas.

Silva Filho e Katsurayama (2008), Parente e Albuquerque (2008) e Zanetti (2008) descreveram o uso de retorno do investimento (ROI) como um forte fator influente na manutenção de um programa de melhoria.

Zanetti (2008) afirma que, durante a execução dos processos, a equipe sentiu, claramente, a necessidade de um mecanismo para reconhecer os benefícios associados aos esforços de melhoria e para motivar os membros no contínuo aperfeiçoamento dos processos, com o objetivo de alcançar níveis mais altos de maturidade. Parente e Albuquerque (2008) também aconselharam as organizações a analisarem o retorno do investimento realizado com a melhoria de processos de software, tornando-os visíveis, pois diminui o risco de interromper o processo de melhoria contínua.

Outros fatores encontrados foram: o montante de recursos disponíveis para a manutenção e a melhoria do processo, e o compromisso dos gestores com o programa de melhoria. De acordo com Parente e Albuquerque (2008), uma empresa que quer melhorar continuamente seus processos precisa fornecer os recursos necessários, pois depende muito do investimento em recursos humanos capacitados, ferramentas de apoio, bem como em consultorias especializadas. Dessa forma, a existência de uma alta direção comprometida, fornecendo o apoio adequado, auxilia a empresa a atingir uma melhoria contínua de seus processos (Monteiro et al., 2008).

Yoshida e Tavares (2008) disseram que a capacidade do SEPG (Software Engineering Process Group) da empresa para pôr em prática o modelo de melhoria afeta diretamente o desempenho do projeto. Em algumas empresas, constatou-se que as atitudes do SEPG podem dificultar o monitoramento das atividades relacionadas à 
implementação e à manutenção da execução dos processos (Yoshida e Tavares (2008), Parente e Albuquerque (2008) e Montoni et al. (2008).

O nível de formalização da comunicação é outro fator descrito por Parente e Albuquerque(2008) e Porto et al. (2008). Já Yoshida e Tavares(2008) disseram que, quão maior o nível de organização da comunicação na empresa, menor o risco de falha na interpretação e na compreensão dos requisitos para a execução das atividades.

Existem também dois fatores que têm semelhanças e são citados por Parente e Albuquerque (2008), Porto et al. (2008) e Rocha et al. (2008), que são: o nível de conhecimento sobre os processos de software e qualidade de software, e o nível de utilização de metodologias e conceitos de engenharia de software.

Para Yoshida e Tavares (2008), um elevado nível de conhecimento e compreensão dos colaboradores, principalmente os formadores de opinião, sobre conceitos relacionados à qualidade de software, é muito importante para avaliar e compreender o impacto que um Programa de Melhoria pode representar para a organização. Quanto maior o nível de conhecimento sobre conceitos relacionados à qualidade de software e ao modelo de maturidade, menor o risco de subestimar os recursos necessários ao programa de melhoria. Montoni et al. (2008) disseram que a implementação de processos de software envolve atividades de conhecimentos especificos. Isso significa que as pessoas envolvidas em iniciativas de melhoria de processos devem ter profundo conhecimento de engenharia de software e devem ser capazez de usar esse conhecimento para orientar a execução dos processos.

A empresa que possui conhecimento sobre qualidade de software e engenharia de software, distribuídos entre seus empregados, tem uma melhor chance de manter suas atividades aderente ao modelo de matridade.

Outro fator que influencia no desejo de realizar as atividades como elas foram executadas antes da avaliação da organização é o nível de resistência às mudanças culturais. A zona de conforto que existe antes da implementação dos processos e durante a manutenção do Programa de Melhoria é uma barreira muito grande.

O ser humano tem uma resistência natural para mudanças. Sempre que a organização deixa a zona de conforto, alguns funcionários se opõem à execução de tarefas necessárias e ao uso de métodos e técnicas exigidas pelo modelo de melhoria.

Porto et al. (2008) e Monteiro et al. (2008) destacaram outro fator importante: o nível de investimento na formação em processos de software. Yoshida e Tavares (2008), por exemplo, disseram que as dificuldades relacionadas com as mudanças culturais podem ser mitigadas com treinamentos, palestras e reuniões em disciplinas complementares.

Um investimento contínuo na formação dos profissionais facilita a continuidade da adesão aos modelos de maturidade, melhorando o conhecimento relacionado à qualidade de software e à engenharia de software.

Um fator que pode dificultar os benefícios das iniciativas de formação profissional é a alta rotatividade dos recursos humanos, porque gera altos custos e o conhecimento não pode ser usado para apoiar o Programa de Melhoria (Porto et al., 2008). 
Sendo que Yoshida e Tavares (2008) defenderam que um Programa de Melhoria deva ser tratado como um projeto, logo, deve ser planejado e monitorado como qualquer projeto de desenvolvimento de software, incluindo a definição de marcos e acompanhamento constante da evolução e dos custos envolvidos nesta iniciativa.

Outro fator encontrado na literatura é a importância dos consultores para obter e manter o processo de software aderente ao modelo de maturidade. Para Porto et al. (2008), a importância dos consultores se torna clara, porque como muitos deles têm a oportunidade de participar de outras avaliações de empresas, como um consultor ou como um avaliador, então eles sabem, exatamente, o exigido e a realidade de outras empresas (benchmarking). Eles observaram que uma empresa de consultoria bem qualificada facilita a definição de metas de melhores negócios para a empresa e uma maior aderência ao modelo de maturidade.

No entanto, deve-se ressaltar que a continuidade do Programa de Melhoria depende de como os consultores são capazes de ensinar aos colaboradores a executar suas atividades e a conscientizá-los de como o conhecimento torna-se um verdadeiro trunfo da organização, ou seja, os consultores podem sair, mas a cultura do processo de software permanece na empresa.

Finalmente, outro fator foi o nível de utilização de ferramentas de apoio. Quanto maior o apoio de ferramentas, menor é o risco de negligenciar as práticas exigidas pelo modelo de maturidade (Yoshida e Tavares, 2008; Silva Filho e Katsurayama , 2008).

\section{Pesquisa Quantitativa Utilizando Survey}

\subsection{Metodologia de Pesquisa do Survey}

O próximo passo da pesquisa consistiu na realização de um survey para identificar o nível de influência dos fatores, identificados na revisão da literatura, na manutenção da aderência do processo de software ao modelo de maturidade.

Além disso, outro objetivo deste estudo foi descobrir os resultados esperados relacionados aos processos exigidos no nível G, o mais simples do MPS.BR, com maior dificuldade de serem mantidos após a avaliação.

Foi definido um questionário dividido em três partes: a primeira seção era um conjunto de questões objetivas, que buscavam caracterizar o profissional respondente; a segunda parte listava os fatores que influenciam a manutenção dos processos de software de acordo com a revisão de literatura e foi perguntado aos respondentes quão relevantes foram os fatores para a manutenção e a execução do Programa de Melhoria. Os entrevistados podiam acrescentar outros fatores que considerassem relevantes. Finalmente, a terceira parte do questionário apresentava os resultados esperados (práticas) dos processos avaliados no nível G do MPS.BR, que são o Gerencimento de Projetos e o Gerenciamento de Requisitos, buscando saber quais eram os mais difíceis de serem continuados após a avaliação ter sido realizada. Pediu-se aos participantes que justificassem a sua avaliação sempre que considerassem importante a sua resposta.

A população dessa pesquisa foi todos os profissionais, certificados ou não, que participaram da execução ou avaliação de processos MPS.BR.

O questionário obteve 27 respostas e teve como amostra de sua população o seguinte perfil: $44 \%$ dos respondentes são mestres e $22 \%$ possuem grau superior 
completo; $40 \%$ tem de 6 a 9 anos de experiência em qualidade de software e $30 \%$ tem de 3 a 6 anos; $48 \%$ já haviam sido gerentes de projetos em 5 ou mais projetos e outros $28 \%$ deles haviam gerenciado de 3 a 5 projetos; $37 \%$ trabalham com gerenciamento de projetos de 1 a 3 anos e outros 25\% de 3 a 6 anos; 66\% deles já participaram de um grupo de processo avaliado; $40 \%$ são implementadores certificados, $33 \%$ são consultores e $29 \%$ são avaliadores certificados do MPS.BR; 50\% dos respondentes participaram de uma ou 2 avaliações e outros $30 \%$ participaram de 5 ou mais.

\subsection{Análise dos Resultados do Survey}

Nessa seção, serão apresentados os resultados dos questionários, divididos em três partes. A primeira delas mostrará os resultados dos fatores dados como influentes na manutenção encontrados na literatura. A segunda parte apresenta as dificuldades de continuidade relacionadas aos resultados esperados do processo Gerência de Projetos (GPR) do MPS.BR. E por fim, serão mostradas as dificuldades em manter os resultdados esperados do processo Gerência de Requisitos (GRE), também do MPS.BR.

Os fatores encontrados na literatura foram analisados usando a seguinte escala de relevância: 0 - Sem relevância; 1 - Baixa importância; 2 - Razoável relevância; 3 Muito importante; 4 - Indispensável.

Tabela 1. Nível de Influencia dos Fatores encontrados na Literatura

\begin{tabular}{|c|c|c|c|c|c|}
\hline Fatores & $\mathbf{0}$ & 1 & 2 & 3 & 4 \\
\hline $\begin{array}{l}\text { F01-Conhecimento da estrutura organizacional } \\
\text { pelos colaboradores }\end{array}$ & $3,7 \%$ & $18,5 \%$ & $33,3 \%$ & $25,9 \%$ & $18,5 \%$ \\
\hline F02- Estrutura eficaz para comunicação & $0 \%$ & $0 \%$ & $22,2 \%$ & $29,6 \%$ & $48,1 \%$ \\
\hline $\begin{array}{l}\text { F03-Alto nível de conhecimento sobre os conteúdos } \\
\text { relacionados ao programa de melhoria de software }\end{array}$ & $0 \%$ & $0 \%$ & $34,6 \%$ & $38,4 \%$ & $26,9 \%$ \\
\hline $\begin{array}{l}\text { F04-Alto nível de maturidade da cultura } \\
\text { organizacional sobre o uso de técnicas e métodos de } \\
\text { engenharia de software }\end{array}$ & $0 \%$ & $0 \%$ & $18,5 \%$ & $44,4 \%$ & $37,1 \%$ \\
\hline $\begin{array}{l}\text { F05-Alto nível do uso de ferramentas de automação } \\
\text { utilizadas no suporte aos processos de software }\end{array}$ & $0 \%$ & $11,1 \%$ & $14,8 \%$ & $37,1 \%$ & $37,1 \%$ \\
\hline F06- Baixo nível de resistência a mudança & $0 \%$ & $0 \%$ & $18,5 \%$ & $44,4 \%$ & $37,1 \%$ \\
\hline $\begin{array}{l}\text { F07- Comprometimento dos gerentes de projeto } \\
\text { com o programa de melhoria }\end{array}$ & $0 \%$ & $0 \%$ & $7,4 \%$ & $11,1 \%$ & $81,4 \%$ \\
\hline $\begin{array}{l}\text { F08-Utilização de indicadores que demonstram o } \\
\text { retorno obtido com a execução do processo }\end{array}$ & $3,7 \%$ & $0 \%$ & $14,8 \%$ & $48,1 \%$ & $33,3 \%$ \\
\hline $\begin{array}{l}\text { F09-Provisão dos recursos necessários pela alta } \\
\text { gerencia }\end{array}$ & $0 \%$ & $0 \%$ & $0 \%$ & $40,7 \%$ & $59,2 \%$ \\
\hline $\begin{array}{l}\text { F10-Utilização de abordagens e ferramentas de } \\
\text { gestão do conhecimento }\end{array}$ & $0 \%$ & $18,5 \%$ & $59,2 \%$ & $22,2 \%$ & $0 \%$ \\
\hline F11- Orientação de uma consultoria externa & $0 \%$ & $7,6 \%$ & $26,9 \%$ & $50 \%$ & $15,3 \%$ \\
\hline $\begin{array}{l}\text { F12-Eficacia da consultoria, que ajuda a empresa a } \\
\text { implementar o processo de software em transformar } \\
\text { o SEPG(Software Engineering Process Group) em } \\
\text { um grupo autônomo e com alto nível de } \\
\text { conhecimento }\end{array}$ & $0 \%$ & $0 \%$ & $7,4 \%$ & $48,1 \%$ & $44,4 \%$ \\
\hline
\end{tabular}




\begin{tabular}{|l|l|l|l|l|l|l|}
\hline F13-Baixo nível de rotatividade de pessoal & $0 \%$ & $0 \%$ & $44,4 \%$ & $44,4 \%$ & $11,1 \%$ \\
\hline $\begin{array}{l}\text { F14-Manutenção de uma política eficaz de } \\
\text { treinamentos }\end{array}$ & $0 \%$ & $3,7 \%$ & $29,6 \%$ & $37,4 \%$ & $29,6 \%$ \\
\hline
\end{tabular}

De acordo com a Tabela 1, os fatores "F07-Compromisso dos gerentes de projeto com o programa de melhoria", "F09- Provisão dos recursos necessários pela alta gerencia", "F02- Estrutura eficaz para comunicação" e "F12-Eficácia da consultoria, que ajuda a empresa a implementar o processo de software em transformar $o$ SEPG(Software Engineering Process Group) em um grupo autônomo e com alto nível de conhecimento" foram considerados os mais relevantes para a continuidade dos processos de software, após a avaliação. Esse resultado corrobora com as conclusões da revisão da literatura.

Entretanto, os fatores "F01- Conhecimento da estrutura organizacional pelos colaboradores" e "F10- Utilização de abordagens e ferramentas de gestão do conhecimento" foram considerados os menos importantes para as necessidades de manutenção. Este resultado não reforçou os achados na revisão da literatura.

A ultima parte do questionário objetivou avaliar os resultados esperados (práticas) dos processos do nível G do MPS.BR. É importante ressaltar que os resultados esperados do processo Gerência de Projetos são semelhantes aos objetivos específicos das áreas de processo Planejamento de Projeto e Monitoramento e Controle de Projetos do CMMI-DEV. Além disso, o processo Gerência de Requisitos do MPS.BR é semelhante à área de processo Gerência de Requisitos do CMMI-DEV (CMU/SEI, 2006).

Assim como dito acima, cada resultado esperado foi avaliado em relação ao nível de dificuldade em continuá-lo depois de uma avaliação MPS.BR. Para isso, a escala utilizada foi: 1 - Dificuldade em continuar; 2 - Dificuldade razoável em continuar; 3 - Fácil de continuar.

A Tabela 2 apresenta os resultados obtidos para os resultados esperados de Gerência de Projetos.

Tabela 2. Resultados para GPR

\begin{tabular}{|l|l|l|l|}
\hline Resultados esperados & $\mathbf{1}$ & $\mathbf{2}$ & $\mathbf{3}$ \\
\hline GPR01 - O escopo do trabalho para o projeto é definido; & $0 \%$ & $6 \%$ & $94 \%$ \\
\hline $\begin{array}{l}\text { GPR02 - As tarefas e os produtos de trabalho do projeto são } \\
\text { dimensionados utilizando métodos apropriados }\end{array}$ & $6 \%$ & $50 \%$ & $44 \%$ \\
\hline GPR03 - O modelo e as fases do ciclo de vida do projeto são definidos & $0 \%$ & $13 \%$ & $88 \%$ \\
\hline $\begin{array}{l}\text { GPR04 - esforço e o custo para a execução das tarefas e dos produtos } \\
\text { de trabalho são estimados com base em dados históricos ou referências } \\
\text { técnicas }\end{array}$ & $50 \%$ & $19 \%$ & $31 \%$ \\
\hline $\begin{array}{l}\text { GPR05 - O orçamento e o cronograma do projeto, incluindo a definição } \\
\text { de marcos e pontos de controle, são estabelecidos e mantidos }\end{array}$ & $25 \%$ & $44 \%$ & $31 \%$ \\
\hline $\begin{array}{l}\text { GPR06 - Os riscos do projeto são identificados e o seu impacto, } \\
\text { probabilidade de ocorrência e prioridade de tratamento são determinados } \\
\text { e documentados }\end{array}$ & $25 \%$ & $38 \%$ & $38 \%$ \\
\hline GPR07 - Os recursos humanos para o projeto são planejados & $19 \%$ & $56 \%$ & $25 \%$ \\
\hline
\end{tabular}




\begin{tabular}{|c|c|c|c|}
\hline considerando o perfil e o conhecimento necessários para executá & & & \\
\hline $\begin{array}{l}\text { GPR08 - Os recursos e o ambiente de trabalho necessários para executar } \\
\text { o projeto são planejados }\end{array}$ & $6 \%$ & $44 \%$ & $50 \%$ \\
\hline $\begin{array}{l}\text { GPR09 - Os dados relevantes do projeto são identificados e planejados } \\
\text { quanto à forma de coleta, armazenamento e distribuição. Um mecanismo } \\
\text { é estabelecido para acessá-los, incluindo, se pertinente, questões de } \\
\text { privacidade e segurança }\end{array}$ & $38 \%$ & $31 \%$ & $31 \%$ \\
\hline $\begin{array}{l}\text { GPR10 - Um plano geral para a execução do projeto é estabelecido com a } \\
\text { integração de planos específicos }\end{array}$ & $6 \%$ & $19 \%$ & $75 \%$ \\
\hline $\begin{array}{l}\text { GPR11 - A viabilidade de atingir as metas do projeto, considerando as } \\
\text { restrições e os recursos disponíveis, é avaliada. Se necessário, ajustes são } \\
\text { realizados }\end{array}$ & $25 \%$ & $38 \%$ & $38 \%$ \\
\hline $\begin{array}{l}\text { GPR12 - O Plano do Projeto é revisado com todos os interessados e o } \\
\text { compromisso com ele é obtido }\end{array}$ & $25 \%$ & $31 \%$ & $44 \%$ \\
\hline $\begin{array}{l}\text { GPR13 - O projeto é gerenciado utilizando-se o Plano do Projeto e outros } \\
\text { planos que afetam o projeto e os resultados são documentados }\end{array}$ & $13 \%$ & $44 \%$ & $44 \%$ \\
\hline GPR14 - O envolvimento das partes interessadas no projeto é gerenciado & $19 \%$ & $44 \%$ & $38 \%$ \\
\hline $\begin{array}{l}\text { GPR15 - Revisões são realizadas em marcos do projeto e conforme } \\
\text { estabelecido no planejamento }\end{array}$ & $6 \%$ & $69 \%$ & $25 \%$ \\
\hline $\begin{array}{l}\text { GPR16 - Registros de problemas identificados e o resultado da análise de } \\
\text { questões pertinentes, incluindo dependências críticas, são estabelecidos e } \\
\text { tratados com as partes interessadas }\end{array}$ & $25 \%$ & $38 \%$ & $38 \%$ \\
\hline $\begin{array}{l}\text { GPR17 - Ações para corrigir desvios em relação ao planejado e para } \\
\text { prevenir a repetição dos problemas identificados são estabelecidas, } \\
\text { implementadas e acompanhadas até a sua conclusão }\end{array}$ & $25 \%$ & $38 \%$ & $38 \%$ \\
\hline
\end{tabular}

Os resultados esperados "GPR4 - O esforço e o custo para a execução das tarefas e dos produtos de trabalho são estimados com base em dados históricos ou referências técnicas" e "GPR9 - Os dados relevantes do projeto são identificados e planejados quanto à forma de coleta, armazenamento e distribuição. Um mecanismo é estabelecido para acessá-los, incluindo, se pertinente, questões de privacidade e segurança” foram considerados como os mais difíceis de serem realizados após a avaliação.

De acordo com um dos participantes, o GPR4 é difícil de ser continuado, porque a manutenção de uma base de dados com um histórico de dados de confiança é um grande desafio e é fundamental para esse resultado esperado. A dificuldade de manter tal base de dados pode fazer os colaboradores envolvidos usarem apenas opiniões subjetivas para estimar o esforço do projeto, influenciando, consequentemente, no custo.

O resultado obtido com a survey para os resultados esperados do processo Gerência de Requisitos (GRE) é apresentado na Tabela 3. 
Tabela 3. Resultados da prática de GRE

\begin{tabular}{|l|l|l|l|}
\hline Prática (Resultados esperados) & $\mathbf{1}$ & $\mathbf{2}$ & $\mathbf{3}$ \\
\hline $\begin{array}{l}\text { GRE01 - Os requisitos são entendidos, avaliados e aceitos junto aos } \\
\text { fornecedores de requisitos, utilizando critérios objetivos }\end{array}$ & $0 \%$ & $38 \%$ & $63 \%$ \\
\hline $\begin{array}{l}\text { GRE02 - O comprometimento da equipe técnica com os requisitos } \\
\text { aprovados é obtido }\end{array}$ & $19 \%$ & $25 \%$ & $56 \%$ \\
\hline $\begin{array}{l}\text { GRE03 - A rastreabilidade bidirecional entre os requisitos e os produtos } \\
\text { de trabalho é estabelecida e mantida }\end{array}$ & $56 \%$ & $38 \%$ & $6 \%$ \\
\hline $\begin{array}{l}\text { GRE04 - Revisões em planos e produtos de trabalho do projeto são } \\
\text { realizadas visando a identificar e corrigir inconsistências em relação aos } \\
\text { requisitos }\end{array}$ & $25 \%$ & $38 \%$ & $38 \%$ \\
\hline \begin{tabular}{l} 
GRE05 - Mudanças nos requisitos são gerenciadas ao longo do projeto \\
\hline
\end{tabular} & $31 \%$ & $38 \%$ & $31 \%$ \\
\hline
\end{tabular}

O resultado esperado "GRE3 - A rastreabilidade bidirecional entre os requisitos e os produtos de trabalho é estabelecida e mantida" foi considerado um dos três resultados mais difíceis em serem continuados pelos respondentes. Para um deles, um consultor, isto deve-se à falta de ferramentas automatizadas que possam garantir a rastreabilidade bidirecional. As ferramentas podem facilitar um pouco mais a continuidade dessa prática.

\section{Pesquisa Qualitativa Utilizando Grounded Thoery}

$\mathrm{Na}$ busca de validar os dados encontrados na pesquisa quantitativa, e de capturar e representar melhor os conhecimentos sobre os fatores que influenciam na continuidade dos processos estabelecidos após a avaliação, foi conduzida uma pesquisa qualitativa, que se baseia nos passos sugeridos por Strauss e Corbin (1998) para a Grounded Theory (GT).

Visando facilitar o entendimento de como foi feita essa pesquisa, a seção 4.1 busca mostrar os principais conceitos da GT e a aplicação deles nessa pesquisa. Já a seção 4.2 se atém a discutir os resultados obtidos na pesquisa qualitativa.

\subsection{Metodologia de Pesquisa Grounded Theory}

Apesar da Grounded Theory ter sido originada nos anos 60 e ter sido extensivamente aplicada nas áreas de ciências sociais, o uso da GT em estudos na área de desenvolvimento de software não é muito comum, sendo geralmente restrita a investigar questões tecnológicas na área de Sistemas de Informação. A aplicação da Grounded Theory nas áreas de engenharia de software e melhoria de processo é ainda mais escassa, como diz Montoni e Rocha (2010), porém alguns trabalhos têm se destacado, assim como esse citado.

Grounded Theory (ou Teoria Fundamentada nos Dados) é um método de pesquisa qualitativo que utiliza um conjunto de procedimentos sistemáticos de coleta e análise dos dados para gerar, elaborar e validar teorias substantivas sobre fenômenos, essencialmente sociais, ou processos sociais abrangentes, segundo Bandeira-de-Melo e Cunha (2006). Seus autores, Glauser e Strauss, afirmam que existem dois tipos básicos de teorias: as formais e as substantivas, como dizem Bianchi e Ikeda (2006). O primeiro tipo é composto das teorias conceituais e abrangentes, enquanto que o segundo tipo é 
específico para determinado grupo ou situação e não visa generalizar além da sua área substantiva.

O método Grounded Theory surgiu a partir de uma publicação de Glaser e Strauss em 1967. Seus criadores divergiram sobre alguns pontos, e o método dividiu-se em duas vertentes. Uma delas é defendida por Glaser (1992) e enfatiza a característica emergente do método e os processos indutivos desenvolvidos pioneiramente pelo Departamento de Sociologia da Universidade de Columbia nos anos 50 e 60. A outra linha foi desenvolvida por Strauss (1987) e consolidada em (Strauss e Corbin, 1998), com o objetivo de sistematizar o método de coleta e análise de dados.

Segundo a linha proposta por Strauss, GT (Grounded Theory) é baseada na idéia de codificação (coding), que é o processo de analisar os dados. Durante a codificação, são identificados conceitos (ou códigos) e categorias. Um conceito (ou código), que tem um significado para o pesquisador, dá nome a um fenômeno de interesse para o pesquisador, abstrai um evento, objeto, ação, ou interação (Strauss e Corbin, 1998). Categorias são agrupamentos de conceitos unidos em um grau de abstração mais alto.

O processo de codificação pode ser dividido em três fases: codificação aberta, axial e seletiva. A codificação aberta envolve a quebra, a análise, a comparação, a conceituação e a categorização dos dados. Segundo Bandeira-de-Melo e Cunha (2006), nas fases iniciais da codificação aberta, o pesquisador explora os dados, examinando minuciosamente aquilo que the parece relevante devido à leitura intensiva dos textos. $\mathrm{Na}$ fase de codificação aberta, os incidentes ou eventos são agrupados em códigos através da comparação incidente-incidente.

Os códigos gerados podem ser classificados como: códigos de primeira ordem, diretamente associados às citações (chamados códigos in vivo); e códigos abstratos ou teóricos, associados a outros códigos, sem, necessariamente, estarem ligados a alguma citação. Também na codificação aberta, é realizada a criação de categorias que agregam os códigos para reduzir o número de unidades com que o pesquisador irá trabalhar (Conte, 2009).

Após a identificação de categorias conceituais pela codificação aberta, a codificação axial examina as relações entre as categorias que formam as proposições da teoria substantiva (Bandeira-de-Melo e Cunha, 2006). Explicitam-se causas e efeitos, condições intervenientes e estratégias de ação, em proposições que devem ser testadas novamente nos dados. As relações entre os códigos podem ser definidas pelo próprio pesquisador. Na linha proposta por Strauss e Corbin (1998), essas relações formam o que os autores denominam de paradigma: condições causais, intervenientes, conseqüências e estratégias de ações/interações.

Por fim, a codificação seletiva refina todo o processo, identificando a categoria central da teoria, com a qual todas as outras estão relacionadas. A categoria central (core category) deve ser capaz de integrar com as demais e expressar a essência do processo social que ocorre entre os envolvidos. Esta categoria central pode ser uma já existente, ou uma nova (Conte ,2009).

\subsection{Análise dos Resultados da Grounded Theory}

Nessa pesquisa qualitativa certos procedimentos da GT foram aplicados. Executou-se as três fases do processo de codificação sugeridas pela GT, para que fosse possível 
encontrar o conjunto de teorias que respondessem a questão em estudo: o que influencia na continuidade ou no abandono dos processos aderentes ao modelo após uma avaliação?.

Para a pesquisa, foi construído um questionário subjetivo de oito itens, que foi submetido a membros do SEPG de empresas avaliadas no modelo MPS.BR. Estas são as questões que visavam caracterizar melhor os conhecimentos obtidos nos resultados da pesquisa quantitativa: "Quais as principais dificuldades para manter o processo aderente ao modelo após a avaliação?" "O que acha que pode diminuir o abandono da aderência do processo ao modelo após a avaliação?" "Que fatores/acontecimentos auxiliaram na manutenção da aderência do processo ao modelo?" "Que fatores/acontecimentos dificultaram a manutenção da aderência do processo ao modelo?" "Quais os processos do modelo são mais difíceis de serem mantidos? E porque?" "Quais os resultados esperados do modelo no nível G são mais difíceis de serem mantidos?" "De um modo geral, como você tem visto o Retorno do Investimento feito na melhoria do processo utilizando o modelo de maturidade?" "Quais os pontos positivos e negativos da implementação do Modelo de Maturidade ?" .

A partir das entrevistas feitas com os membros do SEPG de empresas avaliadas, as respostas foram transcritas para documentos e logo se colecionaram as citações dos entrevistados. Não se utilizou "categorias-semente" (seed categories - um conjunto inicial de códigos para começar a codificação), foi-se criando códigos in vivo a partir do texto dos questionários. Em alguns códigos, foram alterados os textos, para que pudessem facilitar a leitura e para que colecionassem mais citações. Essas alterações fizeram com que alguns códigos encontrados se assemelhassem aos fatores críticos de sucesso encontrados em Montoni e Rocha (2010).

Assim como sugere a experiência de Conte (2009), os procedimentos da codificação aberta estimulam a constante criação de novos códigos e a fusão com os códigos existentes, quando novos dados de evidência e interpretação emergem. Os códigos encontrados nos questionários foram agrupados de acordo com as suas propriedades, formando assim conceitos que representam categorias. Estas categorias foram analisadas e subcategorias foram identificadas com o objetivo de proporcionar uma maior clareza sobre o fenômeno em questão. Finalmente, as categorias e subcategorias foram relacionadas entre si, na etapa de codificação axial.

Os passos da codificação aberta e axial se sobrepõem e se unem, devido à interatividade do processo. Os códigos e categorias identificados passaram por sucessivas revisões, sendo que foram produzidos 28 códigos, associados a 3 categorias e 2 subcategorias, todos ficando abaixo de uma categoria central.

A categoria central (core category) se chama "Fatores que influenciam a manutenção" e possui (sendo utilizada a notação "is a part of" como sugerido por Glaser, 1992) as seguintes categorias "Socioculturais", "Técnicos", "Recursos e Comprometimento".

Durante a pesquisa foram encontradas muitas citações que relatavam os resultados de uma empresa com a presença e com a ausência de um mesmo fator. Dessa forma, para não criar duas categorias que denotassem a presença e a ausência dos mesmos nas realidades das empresas, foi decidido agregar essas citações em apenas um fator, que ficaria com o nome referente à presença do mesmo, mas agregaria as citações que relatassem também a sua ausência. Em relação à notação, está sendo usado 
"Evidencia de dificuldade" para quando estão sendo encontradas apenas citações que denotam a influência negativa de um fator referente à manutenção.

Além disso, nas Figuras 1, 2 e 3, os códigos são apresentados seguidos de dois números que representam respectivamente o grau de fundamentação (groundedness) e o de densidade teórica (density) do código. O grau de fundamentação (groundedness) mostra o número de citações com às quais o código está associado. $\mathrm{O}$ grau de densidade teórica (density) mostra o número de relacionamentos do código com outros códigos (Conte, 2009).

Diferentemente da notação "is part of", que foi utilizada para ressaltar que as categorias fazem parte de uma maior (Ex. "Técnicos" is part of "Fatores que influenciam a manutenção"), a notação "is a" será usada quando um código fizer parte de um tipo ou categoria (Ex. "Consultoria ter compreendido a cultura da empresa" is a "Técnicos", pois faz parte da categoria de fatores técnicos).

A Figura 1 apresenta os resultados da categoria "Técnicos".

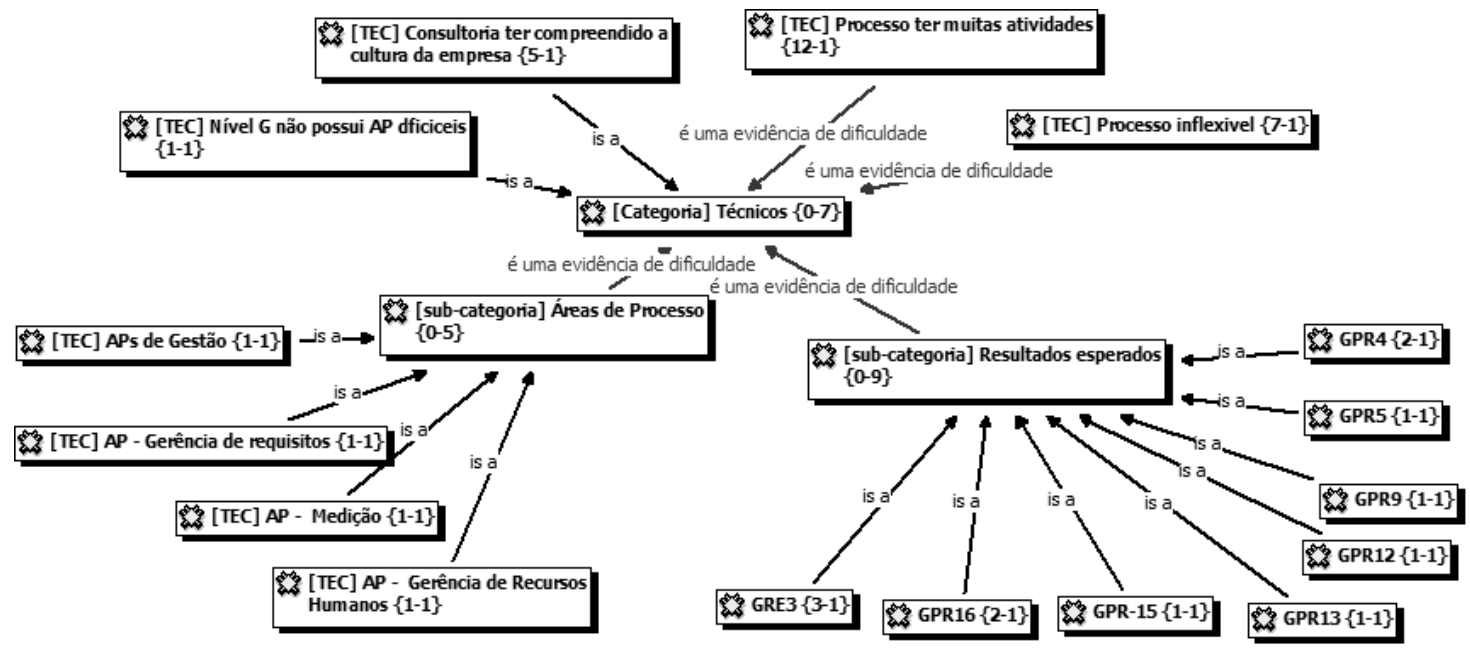

Figura 1. Categoria "Técnicos"

Nessa categoria, os códigos que se destacam são "Processo ter muitas atividades", "Processo Inflexível", que dizem respeito à burocracia e à não inflexibilidade do modelo. Um dos entrevistados disse: "Se fosse um processo mais flexível e não tivesse tantas atividades, seria um processo mais fácil da empresa manter". Outro que se destaca é o código que denota a exigência de uma rastreabilidade bidirecional, pelo modelo no resultado esperado GRE3, confirmando assim o que foi obtido na pesquisa quantitativa.

Outra categoria encontrada na codificação foi a "Sociocultural", que coleciona os fatores Sociais e Culturais, que podem influenciar na manutenção do processo de forma aderente ao modelo (Figura 2). 


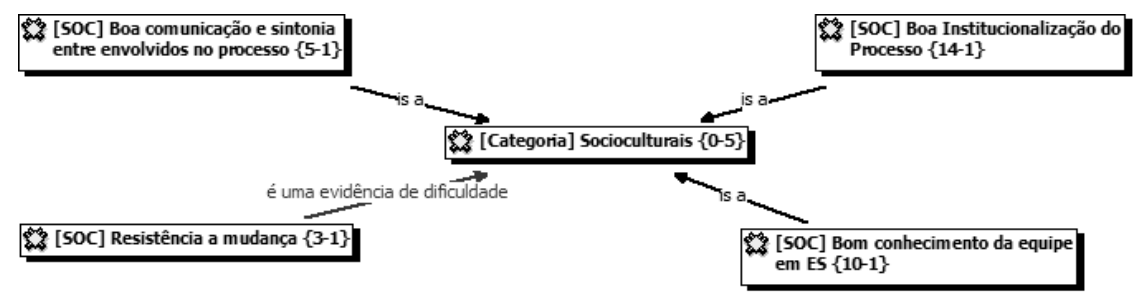

Figura 2. Categoria "Socioculturais"

O código que se destaca nessa categoria é o "Boa Institucionalização do Processo", um dos respondentes comenta que para evitar o abandono dos processos é necessário "Agregar o processo e a gerência de projeto à cultura da empresa."

A última categoria envolve os fatores que citam a necessidade de recursos, sejam pessoais, financeiros ou tempo, ou a necessidade de comprometimento dos membros envolvidos com o processo. A Figura 3 representa a ligação desses códigos.

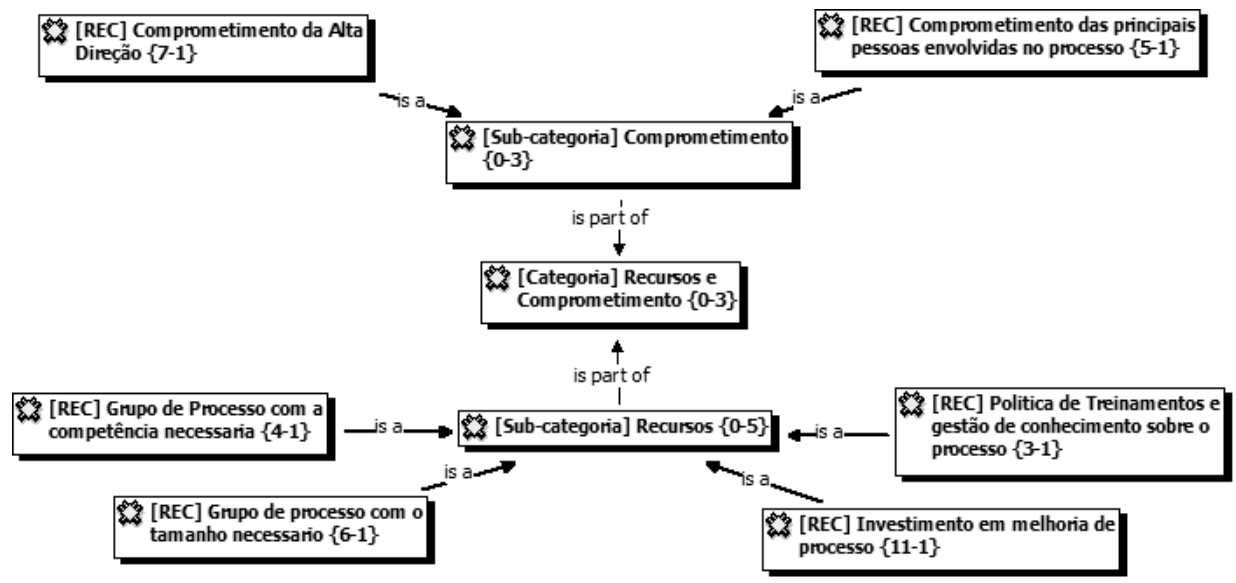

Figura 3. Categoria "Recursos e Comprometimento"

"Comprometimento da Alta Direção" foi o código dessa categoria que obteve mais citações, confirmando a sua importância como fator de influência. Inclusive nas respostas relacionadas a esse fator foi citado por um dos entrevistados que "O comprometimento da alta direção é um dos principais fatores que auxiliam a manutenção".

È interessante analisar os resultados dessa pesquisa qualitativa em relação aos resultados obtidos na pesquisa quantitativa, pois observamos que elas corroboram em muitos fatores.

A quantitativa destacou o "F07- Compromisso de gerentes de projeto com o programa de melhoria" e "F09- Provisão dos recursos necessários pela alta gerencia" corroborando com a qualitativa que destacou o código de "Comprometimento da Alta Direção" e obteve uma boa fundamentação no código "comprometimento das principais pessoas envolvidas no processo".

Outro ponto em que as pesquisas concordaram foi no bom resultado do fator "F12-Eficácia da consultoria, que ajuda a empresa a implementar o processo de software em transformar o SEPG (Software Engineering Process Group) em um grupo autônomo e com alto nível de conhecimento" obtido na pesquisa quantitativa e nos códigos "Boa Institucionalização do Processo" e "Consultoria ter compreendido a cultura da empresa" encontrados na pesquisa qualitativa. 
Com relação aos fatores técnicos, ambas as pesquisas destacam o "GPR4" e o "GRE3" como sendo difíceis de serem continuados. No entanto, elas se diferenciam, pois a pesquisa quantitativa destaca o resultado esperado "GPR9" e a qualitativa dá ênfase ao "GPR16", como difíceis em serem continuados.

\section{Conclusão e Trabalhos Futuros}

Esse trabalho apresentou um resultado de uma revisão da literatura, uma pesquisa quantitativa e uma pesquisa qualitativa referentes às dificuldades que as empresas de software encontram em manter a aderência dos processos de software aos modelos de maturidade, após a avaliação.

Os resultados das duas pesquisas condizem com o que foi achado na literatura, dando mais respaldo a essas referências. Também reafirmam que as empresas de software têm muita dificuldade em continuarem alguns resultados esperados do modelos de maturidade MPS.BR após uma avaliação, que podem ser devido a fatores técnicos, socioculturais ou que envolvem o comprometimento dos interessados para a disponibilização de recursos para essa continuidade.

Um das contribuições deste trabalho é poder ser utilizado por empresas de software, para guiar suas ações relacionadas à manutenção da aderência dos seus processos de software aos modelos de maturidade, mais especificamente ao MPS.BR.

Como trabalhos futuros, pretendemos aumentar o tamanho da amostra e definir um conjunto de ações que possam facilitar a manutenção dos resultados esperados mais difíceis.

\section{Referencias}

Bandeira-de-Melo, R., Cunha, C. (2006) "Grounded Theory". In: Godoi, C. K., Bandeira-de-Mello, R., Silva, A. B. d. (eds), Pesquisa Qualitativa em Estudos Organizacionais: Paradigmas, Estratégias e Métodos, Chapter 8, São Paulo, Saraiva.

Bianchi, E. M. P. G., Ikeda, A. A. (2006) Analisando a Grounded Theory em Administração. IX SEMEAD - Seminários em Administração. São Paulo, Brazil.

CMU/SEI (2006) "Cmmi for development (CMMI-DEV), version 1.2”, technical report CMU/SEI-2006-TR-008. Software engineering institute, Carnegie Mellon University.

Conte, T. (2009) "Técnica de Inspeção de Usabilidade Baseada em Perspectivas de Projeto Web". Tese de Doutorado, COPPE/UFRJ, Rio de Janeiro, RJ, Brasil.

Glaser, B.(1992) Basics of grounded theory analysis. Mill Valley CA, The Sociology Press.

Glaser, B., Strauss, A. (1967) The discovery of grounded theory: Strategies for Qualitative Research. New York, Aldine Transaction

ISO/IEC (2004) International Organization for Standardization and International Electrotechnical Commission. ISO/IEC 12207 Amendment: Information Technology - Amendment 2 to ISO/IEC 12207. Genebra: ISO, 2004

ISO/IEC (2002) "15504, Information Technology - Process Assessment. " 
Kitchenham B. (2004) Procedures for Performing Systematic Reviews. Joint Technical Report Keele University TR/SE-0401 and NICTA Technical Report 0400011T.1, Keele University and NICTA.

Monteiro R.W., Cabral R., Alho F. , Martins, C., Rocha, A.R. (2008) "O esforço requerido para institucionalização de processos de software na Prodepa , Computer engineering system program. Rio de Janeiro, RJ: COPPE/UFRJ.

Montoni, M, Rocha, A.R. (2010) "Aplicação de Grounded Theory para Investigar Iniciativas de Implementação de Melhorias em Processos de Software”. IX Simpósio Brasileiro de Qualidade de Software.

Montoni, M;, Cerdeiral, C., Zanetti, D., Rocha, A.R. (2008) "Uma abordagem para condução de iniciativas de melhoria de processo de software.". Rio de Janeiro, RJ: COPPE/UFRJ.

Parente,T.M.G. , Albuquerque, A.B. (2008) "Domínio informática: a qualidade como foco do seu plano estratégico." SOFTEX IV workshop de Implementadores. IV workshop de consultores MPS.BR, SOFTEX

Pressman, R. S. (2006) "Engenharia de Software” 6ed. São Paulo: McGrow Hill.

Porto, J. B., Pereira, A. C., Pohren J. (2008) "Proposta de Melhoria de Processos da SWB Soluções Integradas usando o MR.MPS e a abordagem PRO2PI", V Workshop de implementadores MPS.BR, SOFTEX.

Rocha, A. R. et al.(2008) "IA COPPE/UFRJ: Lições Aprendidas em 2008", III Workshop de Avaliadores MPS.BR, SOFTEX.

Silva Filho, R. C. e Katsurayama, A. E. (2008) "A experiência na implantação do processo de gerência de reutilização no laboratório de engenharia de software da COPPE/UFRJ " , programa de engenharia de sistemas e computação de Janeiro, RJ: COPPE/UFRJ.

Silva Filho, R. C. (2006) Uma Abordagem para Avaliação de Propostas de Melhoria em Processos de Software. Dissertação de Mestrado, COPPE/UFRJ, Rio de Janeiro, RJ, Brasil.

Softex (2009) "Associação para promoção da excelência do software Brasileiro SOFTEX.” MPS.BR - Guia Geral, versão 1.2. Disponível em: www.softex.br

Strauss, A., Corbin, J. (1998) Basics of Qualitative Research: Techniques and Procedures for Developing Grounded Theory. 2 ed. London, SAGE Publications.

Strauss, A. (1987) Qualitative analysis for social scientists. New York, Cambridge University Press.

Yoshida, D., Tavares, M. (2008) "Lições Aprendidas pela IIITS no Projeto de Implementação MPS.BR Nível G no Grupo de Empresas em Salvador”, SOFTEX IV Workshop de Implementadores

Zanetti, D., Katsurayama, A. E., Santos, G., Montoni, M., Cabral, R., Barreto, A., Rocha A. R. (2008) "Lições aprendidas com a implementação do nível e do MR.MPS no Laboratório de Engenharia de Software da COPPE/UFRJ”, IV Workshop de implementadores MPS.BR 